PROCEEDINGS OF THE

AMERICAN MATHEMATICAL SOCIETY

Volume 137, Number 2, February 2009, Pages 653-662

S 0002-9939(08)09686-X

Article electronically published on September 10, 2008

\title{
CENTER TYPE PERFORMANCE OF DIFFERENTIABLE VECTOR FIELDS IN THE PLANE
}

\author{
ROLAND RABANAL \\ (Communicated by Bryna Kra) \\ Dedicato a Lê Dũng Tráng per il suo sessantesimo compleanno
}

\begin{abstract}
Suppose that $X$ is a planar vector field whose linearization outside some compact set is nonsingular and has pure imaginary spectrum. Then by adding to $X$ a constant vector, one obtains center behavior at infinity: the flow is conjugate to a rotation flow outside some compact set.
\end{abstract}

\section{INTRODUCTION AND STATEMENT OF THE RESUlTS}

In the qualitative theory of planar differential systems, there are many cases where the local phase portrait at a singular point has been characterized (see for instance [21, 5, 16, 2, 7, 8, 20]). On the other hand, we know that some global phase portraits were described in [9, 6]. These theorems may be paraphrased as follows: "the known singular point of $Y$ is a global attractor as long as all the linear parts of $Y$ are asymptotically stable" (see also [17, 4]). This study has motivated the present paper, which is closely related to the results concerning the behavior of a system near infinity [13, 12, 10, 1]. Note that, in order to understand a global phase portrait it is absolutely necessary to research its behavior in a neighborhood of infinity.

For every $\sigma>0$, let $\bar{D}_{\sigma}=\left\{z \in \mathbb{R}^{2}:\|z\| \leq \sigma\right\}$. Thus, $V=\left(\mathbb{R}^{2} \backslash \bar{D}_{\sigma}\right) \cup\{\infty\}$ is the topological subspace of the Riemann sphere on the neighborhood of infinity obtained from $\mathbb{R}^{2} \backslash \bar{D}_{\sigma}$. We consider a differentiable vector field $X: \mathbb{R}^{2} \backslash \bar{D}_{\sigma} \rightarrow \mathbb{R}^{2}$ whose Jacobian determinant $\operatorname{det}(D X)$ is always different from zero, and we denote by $D X_{z}$ the linearization of $X$ at $z \in \mathbb{R}^{2} \backslash \bar{D}_{\sigma}$. In this context, we obtain the following result: "if the eigenvalues of $D X_{z}$ are purely imaginary, then by adding to $X$ some constant $v \in \mathbb{R}^{2}$ one obtains center behavior at infinity, that is: $X+v$ has a periodic trajectory $\Gamma \subset \mathbb{R}^{2} \backslash \bar{D}_{\sigma}$ such that $\Gamma$ is enclosing $\bar{D}_{\sigma}$, and in the unbounded component of $\left(\mathbb{R}^{2} \backslash \bar{D}_{\sigma}\right) \backslash \Gamma$, all the solutions of $X+v$ are periodic trajectories". Notice that the vector field $X:(V, \infty) \rightarrow\left(\mathbb{R}^{2}, 0\right)$ is differentiable in $V \backslash\{\infty\}$, but not necessarily continuous at $\infty$. In the case of global vector fields $Y: \mathbb{R}^{2} \rightarrow \mathbb{R}^{2}$ with $Y(0)=0$, such an eigenvalue condition implies the topological equivalency of $Y$ with the linear vector field $(x, y) \rightarrow(-y, x)$. Observe that as

Received by the editors February 11, 2008.

2000 Mathematics Subject Classification. Primary 34C25; Secondary 34A99.

Key words and phrases. Planar vector fields, periodic solutions, centers, period annulus.

(C)2008 American Mathematical Society 
we study vector fields with nonzero eigenvalues, our assumptions imply that the divergence of the vector field is zero.

The next subsection gives the most basic concepts and notation of the theory of differential equations. This description is necessary because the system $X$ is supposed to be just differentiable (as in our previous papers [6, 10]); thus the eigenvalues of $D X_{p}$ do not depend continuously on $p$. However, in Subsection 2.1, we will see that the solutions of system $X$ are unique as long as $X$ satisfies our eigenvalue condition. In the $C^{1}$ case, the eigenvalue assumption is not necessarily open in the $C^{1}$-Whitney topology. Therefore, a $C^{1}$-vector field whose linearization is nonsingular and has pure imaginary spectrum might not have an approximation by a smooth vector field which also satisfies such eigenvalue conditions.

1.1. Differentiable vector fields. Let $U \subset \mathbb{R}^{2}$ be an open subset, and suppose that $X: U \rightarrow \mathbb{R}^{2}$ is a differentiable vector field. We consider the following autonomous differential equation:

$$
z^{\prime}=X(z)
$$

Since each point on the domain can be an initial condition, such a point jointly with the system (1.1) gives an initial value problem which may have many solutions defined on their maximal interval of existence. Nevertheless, for each of those trajectories - through the same point, kept fixed - all their local funnel sections are compact connected sets (see [14). Moreover, each trajectory has its two limit sets, $\alpha$ and $\omega$ respectively, which are well defined in the sense that they only depend on the solution. Notice that, for us, a trajectory is the curve determined by any solution defined on its maximal interval of existence.

Let $\gamma_{q}$ denote a trajectory passing through a point $q \in U$; thus $\gamma_{q}^{+}$(resp. $\gamma_{q}^{-}$) is the positive (resp. negative) semi-trajectory of $X$ contained in $\gamma_{q}$ and starting at $q$. In this way $\gamma_{q}=\gamma_{q}^{-} \cup \gamma_{q}^{+}$. As usual, a point $p \in U$ in which $X(p)=0$ is called a singular point or a singularity of $X$. When a trajectory $\gamma_{q}$ is defined on $\mathbb{R}$ and there exist $\tau>0$ such that $\gamma_{q}(t+\tau)=\gamma_{q}(t)$ for all $t \in \mathbb{R}$, this $\gamma_{q}$ is said to be a periodic trajectory and such a $q$ is called a periodic point. If $X(p)=0$, this singular point is said to be a center if it admits a punctured neighborhood $\mathcal{A} \backslash\{p\}$ covered with periodic trajectories. The maximal punctured neighborhood of a center is called its period annulus.

Given a differentiable vector field $X: U \rightarrow \mathbb{R}^{2}$, we shall denote by $\operatorname{Spc}(X)=$ \{eigenvalues of $D X_{z}: z \in U$ \}. Our first result is the following.

Theorem A. Let $Y: \mathbb{R}^{2} \rightarrow \mathbb{R}^{2}$ be a differentiable vector field. If $Y(0)=0$ and $\operatorname{Spc}(Y) \subset\{z \in \mathbb{C}: \Re(z)=0\} \backslash\{0\}$, then for any $p \in \mathbb{R}^{2}$ there is a unique trajectory starting at $p$ and the origin is a center whose period annulus is $\mathbb{R}^{2} \backslash\{0\}$.

Theorem A describes the global phase portrait of $Y$ and complements [6, 9, 17].

1.2. Vector fields defined on a neighborhood of infinity. In this subsection we present more vector fields with center behavior. To this end, we denote by $\bar{D}(\Gamma)$ (resp. $D(\Gamma)$ ) the compact disc (resp. open disc) enclosed by a topological circle $\Gamma \subset \mathbb{R}^{2}$.

Definition 1.1. We will say that the differentiable vector field $X: \mathbb{R}^{2} \backslash \bar{D}_{\sigma} \rightarrow \mathbb{R}^{2}$ has a center type performance at infinity if enclosing the origin there exists a periodic trajectory $\Gamma \subset \mathbb{R}^{2} \backslash \bar{D}_{\sigma}$ such that for each $p \in \mathbb{R}^{2} \backslash D(\Gamma)$ : (1) all the solutions $\gamma_{p}$, 
passing through the point $p$, are periodic trajectories and (2) these trajectories also surround the origin, that is, $\bar{D}_{\sigma} \subset \bar{D}(\Gamma) \subset \bar{D}\left(\gamma_{p}\right)$.

The vector fields of Theorem A have center type performance at infinity. We are now ready to state our second result.

Theorem B. Let $X: \mathbb{R}^{2} \backslash \bar{D}_{\sigma} \rightarrow \mathbb{R}^{2}$ be a differentiable vector field. If $\operatorname{Spc}(X) \subset$ $\{z \in \mathbb{C}: \Re(z)=0\} \backslash\{0\}$, then for any $p \in \mathbb{R}^{2} \backslash \bar{D}_{\sigma}$ there exists a unique trajectory starting at $p$. Moreover, there is a constant $v \in \mathbb{R}^{2}$ such that $X+v$ has a center type performance at infinity.

Theorem B complements [12] where the authors study the asymptotic stability at infinity of $C^{1}$-vector fields.

The present paper is organized as follows: Section 2 presents some preparatory results about the behavior of the dynamics at infinity, Section 3 includes the proof of Theorem A, and Section 4 is dedicated to proving Theorem B by using Theorem A.

\section{Preliminary Results}

This section is devoted to some preliminary results which will be used in the proof of the main theorems.

2.1. Uniqueness without the $C^{1}$ condition. The next lemma shows that our eigenvalue condition gives the uniqueness of the trajectories.

Lemma 2.1. If $Y: \mathbb{R}^{2} \rightarrow \mathbb{R}^{2}$ is a differentiable vector field and $\operatorname{Spc}(Y) \subset\{z \in \mathbb{C}$ : $\Re(z)=0\} \backslash\{0\}$, then there exists a unique trajectory of $Y$ starting at $p \in \mathbb{R}^{2}$.

Proof. The assumption over the eigenvalues implies that $\operatorname{Spc}(Y)=\operatorname{Spc}(-Y)$. Since any positive semi-trajectory of $Y$ is a negative semi-trajectory of $-Y$, it is not difficult to obtain the lemma after proving the next affirmation.

(a) For any $p \in \mathbb{R}^{2}$, there is a unique positive semi-trajectory starting at $p$.

Suppose by way of contradiction the existence of some $p \in \mathbb{R}^{2}$ which has at least two positive semi-trajectories $\gamma_{p}^{+} \subset \mathbb{R}^{2}$ and $\sigma_{p}^{+} \subset \mathbb{R}^{2}$ of $Y=(f, g)$. By using the orthogonal vector field $Y^{*}=(-g, f)$, as in [17, we can see that there are $q_{1} \in \gamma_{p}^{+} \backslash\{p\}, q_{2} \in \sigma_{p}^{+} \backslash\{p\}$ and a small compact oriented arc $\left[q_{1}, q_{2}\right]^{*}$ which is tangent to $Y^{*}$ at any point. Moreover, since $Y^{*}(p) \neq 0$ we can assume that $\left\|Y^{*}(z)\right\|>0$ for all $z \in\left[q_{1}, q_{2}\right]^{*}$.

We consider the closed region $B$ whose boundary is the union of $\left[p, q_{1}\right] \subset \gamma_{p}^{+}$, $\left[q_{1}, q_{2}\right]^{*}$ and $\left[p, q_{2}\right] \subset \sigma_{p}^{+}$and apply the Green's formula [18] to the map $z \mapsto Y(z)$. Thus (by using the unitary outer normal vector and the arc length element $d s$, in the line integral) we obtain that

$$
\int_{\left[q_{1}, q_{2}\right]^{*}}\|Y\| d s=\int_{B} \operatorname{Trace}(D Y) d x \wedge d y .
$$

Since the eigenvalue condition shows $\operatorname{Trace}(D Y)=0$, both integrals are zero. This contradiction proves (a) and concludes the proof.

Remark 2.2. This lemma remains true if we consider a differentiable vector field $X: \mathbb{R}^{2} \backslash \bar{D}_{\sigma} \rightarrow \mathbb{R}^{2}$ for which $\operatorname{Spc}(X) \subset\{z \in \mathbb{C}: \Re(z)=0\} \backslash\{0\}$. 
2.2. Pseudo-hyperbolic sector at infinity. In this subsection we describe, in a proposition, some qualitative properties at infinity of the vector field $Y=(f, g)$. To this end, we take $Y^{*}=(-g, f)$ and consider the region $\mathcal{S}=S\left(p_{1}, p_{2} ; q_{1}, q_{2},\left\{\sigma_{i}\right\}\right)$ whose boundary $\partial \mathcal{S}$ is made up of two unbounded semi-trajectories $\left[q_{1}, \infty\right)$ and $\left(\infty, q_{2}\right]$ of $Y$, a compact arc of trajectories $\left[p_{1}, p_{2}\right]$ of $Y$, two $\operatorname{arcs}$ of trajectory $\left[p_{1}, q_{1}\right]^{*},\left[p_{2}, q_{2}\right]^{*}$ of $Y^{*}$, and a set at most countable (which may be empty) of pairwise disjoint trajectories $\sigma_{1}, \sigma_{2}, \ldots, \sigma_{i}, \ldots$ that start and end at infinity.

We call such a region a pseudo-hyperbolic sector of $Y$ if the following conditions are satisfied:

(1) for each $z \in\left[p_{1}, q_{1}\right)^{*}$, there exists an arc of trajectory $[z, \pi(z)) \subset \mathcal{S}$ of $Y$ starting at $z \in\left[p_{1}, q_{1}\right)^{*}$ and ending at $\pi(z) \in\left[p_{2}, q_{2}\right]^{*}$, and

(2) the closure $\bigcup_{z \in\left[p_{1}, q_{1}\right)^{*}}[z, \pi(z)]$ is all of $\mathcal{S}$.

This concept of pseudo-hyperbolic sector at infinity has previously been used in our paper [10] (see also [1, 15]).

Proposition 2.3. Let $Y: \mathbb{R}^{2} \rightarrow \mathbb{R}^{2}$ be a differentiable vector field whose Jacobian determinant is always different from zero. Suppose that:

i) There are constants $s_{1}>0$ and $c>0$ for which $\|z\|>s_{1}$ implies that $\|Y(z)\| \geq c$.

ii) There is $s_{2}>0$ such that for all $z$ with $\|z\|>s_{2}$, the eigenvalues of $D Y_{z}$ are purely imaginary.

Then $Y$ has no pseudo-hyperbolic sector at infinity.

Proof. Suppose by way of contradiction the existence of a pseudo-hyperbolic sector of $Y$. We can change the boundary of the sector and assume that

(a) the pseudo-hyperbolic sector $\mathcal{S}=S\left(p_{1}, p_{2} ; q_{1}, q_{2},\left\{\sigma_{i}\right\}\right)$ is a subset of $\mathbb{R}^{2} \backslash \bar{D}_{s}$, where $s>\max \left\{s_{1}, s_{2}\right\}$, that is, $\mathcal{S} \subset \mathbb{R}^{2} \backslash \bar{D}_{s}$.

Therefore, for any $z \in \mathcal{S}$ we have $\|Y(z)\| \geq c$, and the forward Poincaré map $T:\left[p_{1}, q_{1}\right)^{*} \rightarrow\left[p_{2}, q_{2}\right]^{*}$ is well-defined (subsection 2.1).

(b.1) We claim that, for each $q \in\left[q_{1}, \infty\right)$ there are $p \in\left[p_{1}, p_{2}\right]$ and an arc of trajectory $[p, q]^{*} \subset \mathcal{S}$ of $Y^{*}$ starting from $p$ and ending at $q$.

In order to prove (b.1) we select a sequence $z_{n} \in\left[p_{1}, q_{1}\right]^{*}$ such that $z_{n} \rightarrow q_{1}$ and assume that there are arcs of trajectories $\left[z_{n}, \pi\left(z_{n}\right)\right] \subset \mathcal{S}$ whose union satisfies $\overline{\bigcup_{z_{n} \in\left[p_{1}, q_{1}\right)^{*}}\left[z_{n}, \pi\left(z_{n}\right)\right]}=\mathcal{S}$. From this we define $A_{n}$ as the compact set bounded by the union of the $\operatorname{arcs}\left[p_{1}, z_{n}\right]^{*} \subset\left[p_{1}, q_{1}\right]^{*},\left[z_{n}, \pi\left(z_{n}\right)\right],\left[p_{2}, \pi\left(z_{n}\right)\right]^{*} \subset\left[p_{2}, q_{2}\right]^{*}$ and $\left[p_{1}, p_{2}\right]$.

We consider $\gamma_{q}^{-}$, a negative semi-trajectory of $Y^{*}$ starting at $q \in\left[q_{1}, \infty\right)$. Thus, for some arc $\left[z_{n}, \pi\left(z_{n}\right)\right]$ with $n$ large enough, $\gamma_{q}^{-}$intersects the interior of $A_{n}$. Since $Y^{*}$ is free of singularities in the simply connected set $A_{n} \subset \mathcal{S}$ we obtain (b.1) from the Poincaré-Bendixson Theory [14, p. 156], which claims that there is no semi-trajectory of $Y^{*}$ contained in the compact $A_{n}$. (Notice that, the oriented arc $\left[z_{n}, \pi\left(z_{n}\right)\right]$ is transverse to $\gamma_{q}^{-}$.) Therefore, (b.1) is true.

(b.2) We claim that there exists a constant $K>0$, such that the arc length $\ell\left([p, q]^{*}\right)$ of any $[p, q]^{*}$ is bounded by $K$. That is, for each $[p, q]^{*}$ as in (b.1) we have that $\ell\left([p, q]^{*}\right)<K$. 


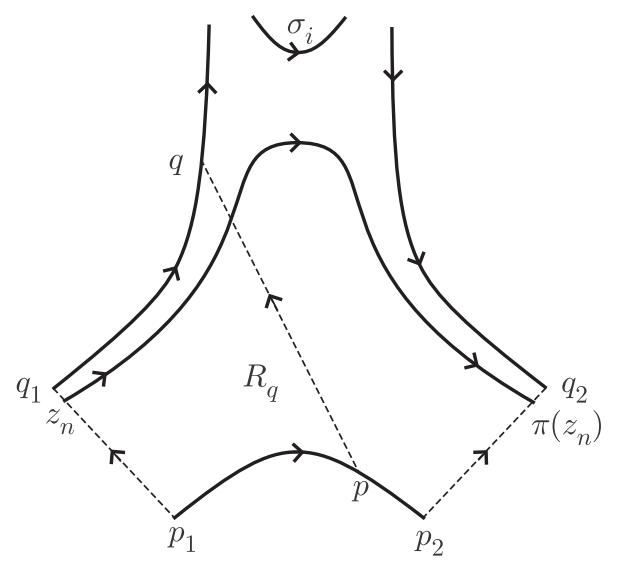

FIGURE 1. Pseudo-hyperbolic sector at infinity

In order to prove (b.2) we denote by $R_{q}$ the compact subset of $\mathcal{S}$ enclosed by the union of the $\operatorname{arcs}\left[p_{1}, q_{1}\right]^{*} \subset \partial \mathcal{S},\left[q_{1}, q\right] \subset\left[q_{1}, \infty\right),[p, q]^{*}$ as in (b.1), and $\left[p_{1}, p\right] \subset$ $\left[p_{1}, p_{2}\right]$ (see Figure 1). By using the Green's formula [18] in $R_{q}$ and the arc length element $d s$, we obtain that

$$
\left|\int_{[p, q]^{*}}\right||Y(s)||d s|=\left|\int_{\left[p_{1}, q_{1}\right]^{*}} \| Y(s)\right||d s| .
$$

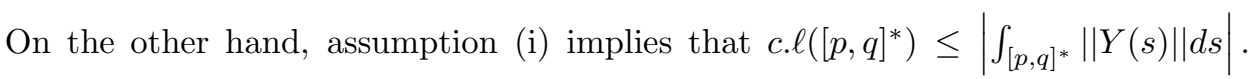
Thus, if we define $d=\max \left\{\|Y(z)\|: z \in\left[p_{1}, q_{1}\right]^{*}\right\}$ the equality of (2.1) shows that

$$
c . \ell\left([p, q]^{*}\right) \leq\left|\int_{\left[p_{1}, q_{1}\right]^{*}}\right||Y(s)||d s| \leq d \ell\left(\left[p_{1}, q_{1}\right]^{*}\right) .
$$

As $c>0$, statement (b.2) holds.

From (b.1) and (b.2) we obtain that the arc $\left[q_{1}, \infty\right)$ is bounded, because the distance from any point $q \in\left[q_{1}, \infty\right)$ to the compact arc $\left[p_{1}, p_{2}\right]$ is smaller than $K$. This contradiction gives the proposition.

Corollary 2.4. For every $Y$ which satisfies the conditions of Proposition 2.3, both vector fields $-Y$ and $Y$ have no pseudo-hyperbolic sector at infinity.

Proof. We refer the reader to Proposition 2.3 .

\section{Centers with global Period annulus}

In this section we present the proof of Theorem A. Thus, we will need the next lemma, which can be deduced from Theorem 2.1 of [1].

Lemma 3.1. If $Y$ is as in Theorem $A$, the map associated to $Y$ is globally injective.

An important consequence of this lemma is that the injective vector fields of Theorem A are free of singularities in a neighborhood of infinity. 
Proposition 3.2. Take $Y: \mathbb{R}^{2} \rightarrow \mathbb{R}^{2}$ to be a local homeomorphism with $Y(0)=0$. If there exists a constant $\sigma>0$ for which the restriction $\left.Y\right|_{\mathbb{R}^{2} \backslash \bar{D}_{\sigma}}$ is differentiable and $\operatorname{Spc}\left(\left.Y\right|_{\mathbb{R}^{2} \backslash \bar{D}_{\sigma}}\right) \subset\{z \in \mathbb{C}: \Re(z)=0\} \backslash\{0\}$, then, for any pair of trajectories $\gamma_{p}$ and $\sigma_{p}$ of $Y$ both contained in $\mathbb{R}^{2} \backslash \bar{D}_{\sigma}$ and passing through the same point $p \in \mathbb{R}^{2} \backslash \bar{D}_{\sigma}$, we obtain that $\gamma_{p}=\sigma_{p}$. Moreover, $Y$ has a center type performance at infinity.

Proof. The uniqueness of the trajectories contained in $\mathbb{R}^{2} \backslash \bar{D}_{\sigma}$ follows directly from Remark 2.2. Therefore we only consider the second affirmation.

By Lemma 3.1] the associated map $Y: \mathbb{R}^{2} \rightarrow \mathbb{R}^{2}$ is injective and $Y(0)=0$. Therefore, we can apply Corollary 2.4 and obtain that:

(a) The injective vector fields $-Y$ and $Y$ have no pseudo-hyperbolic sector at infinity.

From this we can prove the next

ASSERTION: There exists an unbounded sequence of periodic trajectories.

Proof of the assertion. Suppose that $Y$ has no unbounded sequence of periodic trajectories. Thus,

(b.1) there is $s>\sigma$ such that $Y$ has neither singularities nor periodic trajectories in $\mathbb{R}^{2} \backslash \bar{D}_{s}$.

We can apply the results of [14, pp. 166-174] to the (fixed point free) flow induced by $\left.Y\right|_{\mathbb{R}^{2} \backslash \bar{D}_{s}}$ and obtain that for any circle $C \subset \mathbb{R}^{2} \backslash \bar{D}_{s}$ surrounding the origin with a finite number of tangencies, the Brouwer degree $\operatorname{deg}\left(\left.Y\right|_{C}\right)$ satisfies

$$
\operatorname{deg}\left(\left.Y\right|_{C}\right)=\frac{2-n^{e}(Y, C)+n^{i}(Y, C)}{2},
$$

where $n^{e}(Y, C)$ (resp. $\left.n^{i}(Y, C)\right)$ is the number of tangent points of $C$ with $Y$ that are "external" to $D(C)$ (resp. "internal" to $D(C)$ ).

(b.2) We claim that if $C_{s} \subset \mathbb{R}^{2} \backslash \bar{D}_{s}$ minimizes $n^{i}\left(Y, C_{s}\right)$, then every internal tangency in $C_{s}$ gives a pseudo-hyperbolic sector at infinity.

For every internal tangency $q \in C_{s}$ we consider the forward Poincaré map $T$ : $[p, q)_{s} \subset C_{s} \rightarrow C_{s}$ induced by $Y$ (if $T:(q, r]_{s} \subset C_{s} \rightarrow C_{s}$, we obtain a pseudohyperbolic sector of $-Y$ ) where $[p, q)_{s} \subset C_{s}$ is the maximal connected domain of definition of $T$ on which this first return map is continuous.

If the arc of trajectory $(p, \infty) \subset \gamma_{p}^{+}$intersects $C_{s}$, we can apply Lemma 24 and Lemma 25 of 10 , so we can deform $C_{s}$ in a new circle $C^{1} \subset \mathbb{R}^{2} \backslash \bar{D}_{s}$ such that the number of internal tangencies of $C^{1}$ with $Y$ is (strictly) smaller than that of $C_{s}$. This is a contradiction. Therefore $(p, \infty) \subset \gamma_{p}^{+}$is disjoint from $C_{s}$. By using this and our selection of $[p, q)_{s} \subset C_{s}$, it is not difficult to check that there is a pseudo-hyperbolic sector of $Y$ whose boundary intersects $\gamma_{p}^{+} \supset(p, \infty)$. Thus, (b.2) holds.

(b.3) We claim that there exists a circle $C_{s}$ such that $C_{s}$ is transverse to $Y$ and $D_{s} \subset D\left(C_{s}\right)$,

Take a circle $C_{s}$ as in (b.2). By using (a) we have that $\operatorname{deg}\left(\left.Y\right|_{C_{s}}\right)=1$ and $n^{i}\left(Y, C_{c}\right)=0$. Therefore, we obtain (b.3) from the last formula of the Brouwer degree. 
We take the circle of (b.3) and consider the compact disk $\bar{D}\left(C_{s}\right)$. Now we apply the Green's formula of [18] to the map $z \mapsto Y(z)$. Thus

$$
\int_{\bar{D}\left(C_{s}\right)} \operatorname{Trace}(D Y) d x \wedge d y=\oint_{C_{s}}\langle Y(s), \eta(s)\rangle d s,
$$

where $\eta(s)$ is the unitary outer normal vector to $C_{s}$ and $\langle Y(s), \eta(s)\rangle$ is the inner product of $Y(s)$ with $\eta(s)$. Since $C_{s}$ is transverse to $Y$ we obtain

$$
0 \neq \oint_{C_{s}}\langle Y(s), \eta(s)\rangle d s=\int_{\bar{D}\left(C_{s}\right)} \operatorname{Trace}(D Y) d x \wedge d y
$$

But, the eigenvalue assumption shows that $\int_{\bar{D}\left(C_{s}\right)} \operatorname{Trace}(D Y) d x \wedge d y=0$. This contradiction gives the assertion.

In order to conclude this proof we consider the sequence of the assertion. Since $Y(0)=0$ and $Y$ is injective, we obtain that:

(c) There exists an unbounded set of periodic trajectories $\left\{\Gamma, \Gamma_{1}, \Gamma_{2}, \ldots, \Gamma_{n}, \ldots\right\}$ such that $\bar{D}_{\sigma}$ is a proper subset of $\bar{D}(\Gamma)$ and

$$
\bar{D}(\Gamma) \subset \bar{D}\left(\Gamma_{1}\right) \subset \bar{D}\left(\Gamma_{2}\right) \subset \cdots \subset \bar{D}\left(\Gamma_{n}\right) \subset \cdots .
$$

Moreover, we can assume that $\mathbb{R}^{2} \backslash D(\Gamma)$ has no singular points of $Y$.

(d) We claim that the elements of $\mathbb{R}^{2} \backslash D(\Gamma)$ are periodic points.

For each $p \in \mathbb{R}^{2} \backslash D(\Gamma)$ there exists a compact annulus $\bar{A}_{n}=\bar{D}\left(\Gamma_{n}\right) \backslash D(\Gamma)$ containing $p$. Hence, the trajectory starting at $p$ and its two limit sets are contained in $\bar{A}_{n}$, that is, $\gamma_{p} \subset \bar{A}_{n}$ and $\alpha\left(\gamma_{p}^{-}\right) \cup \omega\left(\gamma_{p}^{+}\right) \subset \bar{A}_{n}$. Therefore, the Poincaré-Bendixon Theorem implies that both limit sets $\alpha\left(\gamma_{p}^{-}\right)$and $\omega\left(\gamma_{p}^{+}\right)$are periodic trajectories of $Y=(f, g)$ surrounding $D(\Gamma)$.

To proceed we can assume that $\omega\left(\gamma_{p}^{+}\right)$is clockwise oriented because, in the other case, the construction is similar.

By using the trajectory $\gamma_{q}^{*}$ of $Y^{*}=(-g, f)$ for some $q \in \omega\left(\gamma_{p}^{+}\right)$, it is not difficult to see that there are two arcs of trajectories $\left[p_{1}, p_{2}\right] \subset \gamma_{p}^{+}$and $\left[p_{1}, p_{2}\right]^{*} \subset \gamma_{q}^{*}$ (or $\left[p_{2}, p_{1}\right]^{*} \subset \gamma_{q}^{*}$ when $\gamma_{p}^{+}$surrounds the disk bounded by $\left.\omega\left(\gamma_{p}^{+}\right)\right)$. These two arcs bound a compact set $B \subset \bar{A}_{n}$ where we can apply the Green's formula to the map $z \mapsto Y(z)$. This formula implies that

$$
\left|\int_{\left[p_{1}, p_{2}\right]^{*}}\right||Y(s) \| d s|=0 .
$$

As $\left[p_{1}, p_{2}\right]^{*}$ is free of singularities, we have that $p_{1}=p_{2}$. Therefore, $\gamma_{p}$ is periodic. This implies (d). We conclude this proof from Definition 1.1.

Corollary 3.3. Let $Y: \mathbb{R}^{2} \rightarrow \mathbb{R}^{2}$ be a globally injective local homeomorphism with $Y(0)=0$. Suppose that there is $\sigma>0$ such that $\left.Y\right|_{\mathbb{R}^{2} \backslash \bar{D}_{\sigma}}$ is differentiable and $\operatorname{Trace}\left(\left.D Y\right|_{\mathbb{R}^{2} \backslash \bar{D}_{\sigma}}\right)=0$. Then, the trajectories of $\left.Y\right|_{\mathbb{R}^{2} \backslash \bar{D}_{\sigma}}$ are unique and $Y$ has a center type performance at infinity.

Proof. There are constants $s_{1}>0$ and $c>0$ for which $\|z\|>s_{1}$ implies that $\|Y(z)\| \geq c$. So, Corollary 2.4 shows that the injective vector fields $-Y$ and $Y$ have no pseudo-hyperbolic sector at infinity. Therefore, we obtain this corollary from a slight change in the proof of Proposition 3.2 . 
Before continuing, let us recall that the Local Inverse Function Theorem is also true for differentiable maps whose Jacobian determinant is always different from zero; see [3, 19] and its references.

3.1. Proof of Theorem A. By Proposition 3.2, it suffices to prove that all the elements in $\bar{D}(\Gamma) \backslash\{0\}$ are periodic points, where $\Gamma$ is the periodic trajectory given by Definition 1.1.

(a) We claim that for each $p \in \bar{D}(\Gamma) \backslash\{0\}$, at least one of its limit sets is a periodic trajectory.

Suppose, by contradiction, the existence of some $p \in \bar{D}(\Gamma) \backslash\{0\}$ for which (a) is false. Since the compact set $\bar{D}(\Gamma)$ contains the trajectory at every one of its points, Lemma 3.1 and the Poincaré-Bendixson Theorem imply that $\omega\left(\gamma_{p}^{+}\right)=$ $\alpha\left(\gamma_{p}^{-}\right)=0$. Let $Y=(f, g)$, consider the orthogonal vector field $Y^{*}=(-g, f)$ and a trajectory of $Y^{*}$ starting at $p$, say $\gamma_{p}^{*}$. We take the semi-trajectory of $\gamma_{p}^{*}$ which goes into the compact set bounded by $\gamma_{p} \cup\{0\}$. To proceed we can suppose that this semi-trajectory is the positive one, that is, $\left(\gamma_{p}^{*}\right)^{+}$. Thus the Poincaré-Bendixson Theory [14, p. 151] implies that $\omega\left(\left(\gamma_{p}^{*}\right)^{+}\right)=\{0\}$. Hence, $\gamma_{p}^{-} \cup\left(\gamma_{p}^{+}\right)^{*} \cup\{0\}$ bounds a compact set where we can apply the Green's formula to the map $z \mapsto Y(z)$. This formula implies that

$$
\left|\int_{\left(\gamma_{p}^{*}\right)^{+}} \| Y(s)\right||d s|=0 .
$$

This contradiction proves (a).

In order to conclude the proof we consider $p \in \bar{D}(\Gamma) \backslash\{0\}$. From (a), $\omega\left(\gamma_{p}\right)$ or $\alpha\left(\gamma_{p}\right)$ is periodic. Since $\bar{D}(\Gamma) \backslash\{0\}$ is free of singular points of $Y$ we can proceed as in the proof of (3.1) and obtain that $p$ is periodic. Therefore, Theorem A holds.

Corollary 3.4. Let $Y: \mathbb{R}^{2} \rightarrow \mathbb{R}^{2}$ be a differentiable vector field with $\operatorname{det}(D Y) \neq 0$. Suppose that $Y$ is globally injective, $Y(0)=0$ and Trace $(D Y)=0$. Then the origin is a center whose period annulus is $\mathbb{R}^{2} \backslash\{0\}$.

Proof. We refer the reader to Corollary 3.3 and the proof of Theorem A.

\section{Center type Performance at infinity}

This section is devoted to extending the result of the previous section to a vector field defined on a neighborhood of infinity which can be a proper subset of the plane. To this end, we shall need the following result contained in [11.

Theorem 4.1. Let $X: \mathbb{R}^{2} \backslash \bar{D}_{\sigma} \rightarrow \mathbb{R}^{2}$ be a differentiable vector field. If for some $\varepsilon>0, \operatorname{Spc}(X) \cap(-\varepsilon,+\infty)=\emptyset$, then there exists $s \geq \sigma$ such that $\left.X\right|_{\mathbb{R}^{2} \backslash \bar{D}_{s}}$ can be extended to a globally injective local homeomorphism $\tilde{X}: \mathbb{R}^{2} \rightarrow \mathbb{R}^{2}$.

As an application of this theorem and the results of Section 3 we obtain the next theorem.

Theorem B. Let $X: \mathbb{R}^{2} \backslash \bar{D}_{\sigma} \rightarrow \mathbb{R}^{2}$ be a differentiable vector field. If $\operatorname{Spc}(X) \subset$ $\{z \in \mathbb{C}: \Re(z)=0\} \backslash\{0\}$, then for any $p \in \mathbb{R}^{2} \backslash \bar{D}_{\sigma}$ there exists a unique trajectory starting at $p$. Moreover, there is a constant $v \in \mathbb{R}^{2}$ such that $X+v$ has a center type performance at infinity. 
Proof. Let $\tilde{X}: \mathbb{R}^{2} \rightarrow \mathbb{R}^{2}$ be the topological embedding given in Theorem 4.1 Set $v=-\tilde{X}(0)$ and consider the global injective map $\tilde{X}+v$ which sends the origin into itself. Therefore, $Y:=\tilde{X}+v$ satisfies Proposition 3.2 Since $Y=X+v$ in a neighborhood of infinity, we conclude the proof.

\section{ACKNOWLEDGEMENTS}

This paper was written during a stay of the author at ICTP as a post-doctoral fellow; we wish to thank the members of the Mathematics Section for their kind hospitality. We wish to express our gratitude to the referee, whose comments have been appreciated and incorporated into this work. I am particularly indebted to Carlos Gutiérrez for useful comments on this work.

\section{REFERENCES}

[1] B. Alarcón; V. Guíñez; C. Gutiérrez: Hopf bifurcation at infinity for planar vector fields. Discrete Contin. Dyn. Syst. 17 (2007) 247-258. MR.2257430 (2007g:37035)

[2] M. Berthier; D. Cerveau; A. Lins Neto: Sur les feuilletages analytiques réels et le problème du centre (French). J. Differential Equations 131 (1996) 244-266. MR.1419014 (98a:58128)

[3] A. V. Cernavskii: Addendum to the paper "Finite-to-one open mappings of manifolds". Mat. Sb. (N.S.) 66 (1965) 471-472. MR0220254 (36:3320)

[4] A. Cima; A. van den Essen; A. Gasull; E. Hubbers; F. Mañosas: A polynomial counterexample to the Markus-Yamabe conjecture. Adv. Math. 131 (1997) 453-507. MR.1483974 (98k:34084)

[5] F. Dumortier; R. Roussarie; J. Sotomayor; H. Żoladek: Bifurcations of planar vector fields. Nilpotent singularities and Abelian integrals. Lecture Notes in Math. 1480, Springer-Verlag, Berlin, 1991. MR.1166189 (93f:58165)

[6] A. Fernandes; C. Gutiérrez; R. Rabanal: Global asymptotic stability for differentiable vector fields of $\mathbb{R}^{2}$. J. of Differential Equations 206 (2004) 470-482. MR2096702 (2005h:37055)

[7] A. Gasull; J. Llibre; V. Mañosas; F. Mañosas: The focus-centre problem for a type of degenerate system. Nonlinearity 13 (2000) 699-729. MR1758996 (2001b:34061)

[8] L. Gavrilov: Isochronicity of plane polynomial Hamiltonin systems. Nonlinearity 10 (1997) 433-448. MR:1438261 (98b:58143)

[9] C. Gutiérrez: A solution to the bidimensional global asymptotic stability conjecture. Ann. Inst. H. Poincaré Anal. Non Linéaire 12 (1995) 627-671. MR.1360540 (96k:34099b)

[10] C. Gutiérrez; B. Pires; R. Rabanal: Asymptotic stability at infinity for differentiable vector fields of the plane. J. Differential Equations 231 (2006) 165-181. MR2287882(2008a:34111)

[11] C. Gutiérrez; R. Rabanal: Injectivity of differentiable maps $\mathbb{R}^{2} \rightarrow \mathbb{R}^{2}$ at infinity. Bull. Braz. Math. Soc. (N.S.) 37 (2006) 217-239. MR2266382(2007k:58015)

[12] C. Gutiérrez; A. Sarmiento: Injectivity of $C^{1}$ maps $\mathbb{R}^{2} \rightarrow \mathbb{R}^{2}$ at infinity and planar vector fields. Astérisque 287 (2003) 89-102. MR2040002 (2005c:37028)

[13] C. Gutiérrez; M. A. Teixeira: Asymptotic stability at infinity of planar vector fields. Bull. Braz. Math. Soc. (N.S.) 26 (1995) 57-66. MR.1339178 (96c:58099)

[14] P. Hartman: Ordinary differential equations. Second Edition. Reprinted. Classics in Applied Mathematics 38, SIAM, 2001. MR/1929104(2003h:34001)

[15] X. Jarque; Z. Nitecki: Hamiltonian stability in the plane. Ergodic Theory Dynam. Systems 20 (2000) 775-799. MR1764927 (2001d:37019)

[16] R. Moussu: Symétrie et forme normale des centres et foyers dégénérés (French). Ergodic Theory Dynam. Syst. 2 (1982) 241-251. MR693979 (84j:58090)

[17] C. Olech: On the global stability of an autonomous system on the plane. Contributions to Differential Equations 1 (1963) 389-400. MR0147734 (26:5248)

[18] W. F. Pfeffer: Derivation and integration. Cambridge Tracts in Math. 140, Cambridge, Univ. Press, Cambridge, 2001. MR 1816996 (2001m:26018)

[19] S. Radulescu; M. Radulescu: Local inversion theorems without assuming continuous differentiability. J. Math. Anal. Appl. 138 (1989) 581-590. MR991045 (90e:58007) 
[20] R. Roussarie: Bifurcation of planar vector fields and Hilbert's sixteenth problem. Progr. Math. 164, Birkhäuser Verlag, Basel, 1998.

[21] F. Takens: Singularities of vector fields. Inst. Hautes Études Sci. Publ. Math. 43 (1974) 47-100. MR0339292 (49:4052)

The Abdus Salam International Centre for Theoretical Physics, Trieste, Italy

E-mail address: rrabanal@ictp.it 